homegrown science and technology that could effectively address critical indigenous problems.

Why, then, were universities not turned to as ideal places to study, research, and demonstrate science-based solutions to critical problems? Universities and professors in developing countries bear part of the responsibility for their marginalization. Having trained in universities in the developed world; having pursued dissertation topics of interest to the developed world; having forged joint research projects with colleagues in the developed world; and having defined a successful career by standards set by their counterparts in the developed world, scientists in the developing world-more often than not-found themselves disengaged from their societies.

After more than two decades spent largely exiled in developed countries or as castaways in their own nations, scientists in the developing world are now being welcomed back into their societies. This time, however, governments are insisting that investments in science and technology provide a payoff in terms of improvements in economic and social well-being. And this time, scientists are increasingly recognizing that they need to be responsive to their societies' concerns if the funding is to continue.

This rapprochement between science and society in the developing world has not been easy. Yet many signposts along the way have signaled the advances. These signposts include, for example, Brazil, China, and India's success in promoting science-based development; the growing maturity of university systems in these and other countries that has led institutions of higher education to embrace long-term responsibilities for education, research, and community service; and expanding efforts at South-South cooperation marked not only by exchange programs such as the South-South fellowship program of the Academy of Sciences for the Developing World for postgraduate and postdoctoral research, which provides some 250 fellowships a year, but the China-Brazil Earth Resources Satellite program, begun in 1998 , which has led to the launch of two Earth-imaging satellites, with two more launches planned by 2008. Indeed signposts, both large and small, abound, creating a sense of positive direction and optimism for the future of higher education in the developing world that is unprecedented.

Despite the recent progress, we must examine and evaluate on an ongoing basis what individual scientists and scientific institutions are contributing to society-in terms of improved nutrition, better health, more reliable energy supplies, enhanced communications, a cleaner environment and, perhaps most importantly, overall efforts to break the cycle of extreme poverty that has afflicted too many places in the developing world for decades.

Nevertheless, the key to success, especially for university departments and faculties of science lies in excellence. That's because science without excellence is not science. But success, as I have argued here, also lies in relevance. That's because without responding to the needs of society, university science departments will find it difficult to sustain society's support. Moreover, without educating and training students in ways that make them employable within their own countries, young scientists and technologists will not stay home once they graduate.

As recent history in the developing world shows, successful institutions of higher education without societal purpose will not be successful for long.

This article is reprinted, with permission, from IAU Horizons I2 (34), 2006. (C) International Association of Universities.

\section{Exam Trends and Global Talent Flows}

\section{Johnette Peyton and Veronica A. Garcia}

Johnette Peyton is a manager of applied research at the Graduate Management Admission Council@. Address: 1600 Tysons Boulevard, Suite 1400, McLean, VA 22102, USA. E-mail: jpeyton@gmac.com. Veronica A. Garcia is research writer/editor at the Graduate Management Admission Council@. E-mail:vgarcia@gmac.com.

Tn a recent issue of the Harvard Business Review, Richard Florida identified students as the leading indicator of global talent flow, stating that countries and regions that attract students have an advantage on retaining them and attracting additional pools of talent. If Florida's statement holds true, then new data from the Graduate Management Admission Council (GMAC) appear to demonstrate evidence of this new competitive landscape.

GMAC, a global not-for-profit education organization of leading graduate business schools and owner of the Graduate Management Admission Test (GMAT), publishes Geographic Trend Reports identifying migratory trends among GMAT examinees. Findings are based on voluntary responses to the GMAT background information questionnaire along with score report and registration information in a given testing year. The most recent report data include $2 \mathrm{I} 2,532$ examinee records in 200I and 200,503 in 2005. Approximately twothirds had US addresses at the time of registration in both years.

Test report submissions are used to gauge interest in studying in a particular region, as GMAT scores often accompany applications to graduate business programs. GMAC findings suggest geographic trends for examinees shifted drastically in some regions from 200I to 2005. Data from both years identify a strong preference for US programs overall with a slight decline from $200 \mathrm{I}$ to 2005 , as well as increasing interest in programs located in England, France, India, and Greece. 
However, changes in Greece are primarily linked to increased examinees from Greece.

\section{North Americans Stick Close to Home}

US citizens sent nearly 99 percent of their score reports to programs in the United States both years. Canadians, though slightly more diverse, sent 95 percent of their score reports to programs in North America (about 62 percent to Canadian programs and 32 percent to US programs).

Among US citizens, schools located in England made the greatest gains, and Spain doubled their percentage. Canadians, though consistent in their preference for North America, did show slightly increased interest in England, France, and Hong Kong from one year to the next.

\section{Major Shifts in Western Europe, Central Asia, and the Middle EAST}

Citizens of Western Europe, Central Asia, and the Middle East displayed dramatically reduced interest in US programs between 200I and 2005. (Regional categories by country divide the continent of Asia into two categories: Asia and Central Asia.) Among all citizens of Western Europe, the data show an increasing desire to remain close to home. Programs in Greece experienced the greatest percentage gain among all examinees from Western Europe, moving from sixth to fourth overall.

From citizens of Central Asia, which includes India according to the GMAC regional breakdown of the report, India gained the most market share from the decreased interest in US programs. In addition, citizens of Central Asia displayed increased interest in studying in England, Singapore, and Canada from 2001 to 2005 .

The majority of GMAT examinees from the Middle East region, as defined in the report, are from Israel. Perhaps that explains why examinees from the region place programs located in Israel at the top of their list. Citizens of the Middle East region additionally displayed increased interest in programs located in Canada, Lebanon, and England. Also, the United Arab Emirates, which was not ranked in 200I, ranked seventh in 2005 for Middle Eastern examinees.

\section{Minor Shifts in Eastern Europe, Asia, Africa, and Latin AMERICA}

Test takers from eastern Europe, Asia, Africa, and Latin America consistently prefer US programs, but slight declines were noted from 200 I to 2005 . In eastern Europe, this decline in US programs was countered by increased interest in programs located in England and France.

Asians, who include Chinese, continue to place Canadian programs as a distant second to programs in the United States. In third place, however, England replaced Hong Kong among Asian citizens between the two years, and Thailand, which was not in the top Io in 200I, ranked Ioth among Asian citizens in 2005. Asian citizens also displayed increasing interest in
England, China, France, the Netherlands, and Singapore.

For Africans, England replaced Canada as second behind the United States, and South Africa, Kenya, and Egypt continue to be popular. Declining interest in US programs from citizens of the Mexico/Caribbean/Latin America region was primarily replaced by increasing interest in Canada, England, France, Spain, and the Netherlands. Germany also debuted for the first time among citizens of Mexico/Caribbean/Latin America in 2005 .

\section{Oceania Bucks the Trend}

Contrary to trends noted for all other world regions, citizens of

US business programs, which have long been the primary destination of GMAT examinees, are facing increasing competition from England, France, and India.

Australia, New Zealand, and the Pacific Islands (Oceania, collectively), displayed increased interest in US programs over the years reported, but other countries also witnessed increased interest from this region. The percentages of examinees sending scores to England, France, and Ireland all increased while the percentage of score reports sent to schools in Australia, Hong Kong, and Canada decreased. Interest in New Zealand programs also rose for the Oceania group overall.

\section{Discussion}

US business programs, which have long been the primary destination of GMAT examinees, are facing increasing competition from England, France, and India. Interest in Canadian programs, though consistently second on the list overall, is fluctuating by region, and programs in the Middle East and western Europe are experiencing the greatest increases from their own citizens in recent years.

If these trends are indicative of long-term talent flows, as predicted by Florida, then we may in fact be witnessing subtle shifts in market advantage around the world. A comparison of companies listed in the top Ioo of the Fortune Global 500 list for 2005 shows US companies generated nearly three times the revenue of their nearest competitors combined-in Germany, followed next by those in France, the United Kingdom (particularly England), and Japan. Given the current separation between revenues, it may be difficult for any one area to overtake the lead position in the near term, but there is certainly evidence of opportunity if talent continues to favor a more diverse distribution. And this opportunity will be particularly evident if the population of students educated abroad grows from I.7 million in 200 I to more than 8 million by 2025 , as estimated by UNESCO.

The full report, including regional category descriptions and fur- 
ther information on trends in specific European and Asian countries, is available at www.gmac.com/researchreports.

\section{The Dominance of English in Global Scholarly Publishing}

\section{Mary Jane Curry and Theresa Lillis}

Mary Jane Curry is assistant professor of education at the Warner Graduate School of Education, University of Rochester. Address: PO Box 270425, Dewey Hall, Rochester, NY 14627, USA. E-mail: mjcurry@warner.rochester.edu. Theresa Lillis is senior lecturer in language and communication in the Centre for Language and Communications at the Open University, UK. For more information on their project see www.paw.open.ac.uk.

$\mathbf{T}^{\mathrm{n}}$ an era characterized by globalization, the enterprise of aca1 demic research would ideally capitalize on contributions from scholars all over the world. Yet language barriers can present a considerable obstacle to the global circulation of research findings. The dominance of English as the language of scholarly publishing means that scholars around the world are under increasing pressure to publish their research in English. This situation is problematic in two important ways. First, scholars outside of English-dominant contexts face issues of equity in their access to publishing venues, particularly high-status English-medium research journals. While such scholars experience increasing pressure to publish in English (as a major criterion for promotion and research grants), they often have uneven access to the means to do so, including monies for conference travel and research collaboration, library and other resources, and time to write in English. Second, even as multilingual scholars' material conditions may hinder their English publishing, the global research community suffers from not receiving their research findings, insights, and methodologies. The result may be the emergence of what Polish scholar Anna Duszak calls an "academic monoculture."

\section{The Challenges of Publishing in English}

Since 200 I we have been conducting a study of some 50 scholars in southern and eastern Europe to understand the effects of the dominance of English on global academic knowledge production. Multilingual scholars attempting to publish in
English face a number of challenges, the least of which may be their technical competence in English. Typically, publishing in English entails more than direct translation of academic writing. Rather, a key to scholars' success in publishing is their interactions with "literacy brokers" - gatekeepers such as journal editors and peer reviewers as well as disciplinary and language specialists who may help at various points in the trajectory of writing and publishing research articles.

Gaining access to literacy brokers can be difficult but may happen through participation in local, regional, and international scholarly research networks, whether formal or informal. The most useful types of "brokers" appear to be disciplinary specialists who are attuned to the key research questions, current discussions, and debates of the field and methodologies preferred by linguistic "center"-based journals. However, the interventions of some brokers may result in pressure on multilingual academics to skew their writing to achieve publication by matching the preferences of center-based journals. Our research provides evidence of the relegation of periphery scholars to roles in which they consume and confirm centerbased research but are not allowed access to platforms from which to contribute different perspectives and findings.

\section{The Global Politics of Language}

The global dominance of English in scholarly publishing has implications for international higher education along two main lines: (I) for gatekeepers of scholarly publication and participation in international academic conferences to understand the challenges that multilingual scholars confront; and (2) for national governmental and institutional policymaking bodies to consider the effects of the premium placed on English-medium journal publishing.

First, in terms of the gatekeeping activities of journals and

The dominance of English as the language of scholarly publishing means that scholars around the world are under increasing pressure to publish their research in English.

conferences, it is important for journal reviewers and editors, conference organizers, and proposal reviewers from the English-dominant center to understand the burdens of time, money, and access to research that may hinder multilingual scholars from disseminating their work in English. These constraints may be reflected in submissions that do not reference the most up-to-date literature from English-medium journals, or use nonstandard features of English. The topics and questions that periphery scholars engage with may also not be perceived as "relevant" to current center academic debates because what counts as relevant is often determined by Anglophone center scholars and institutions. Anglophone con- 